\title{
Deep Sedation for Patients with Single Ventricle Physiology
}

\author{
Sarah Abdel-Mageed', Matthew Friedman²
}

${ }^{1}$ Indiana University School of Medicine; ${ }^{2}$ Section of Pediatric Critical Care Medicine, Department of Pediatrics, Riley Hospital for Children at Indiana University Health

\section{Background:}

Advancements in the palliative surgery of patients with single ventricle physiology have led to an increase in lifespan, necessitating the study of safe deep sedation protocols for painful procedures. This study aims to describe the practice of deep sedation by intensivists outside the operating from for children with single ventricle physiology.

\section{Methods:}

A retrospective chart review was conducted on consecutive patients with single ventricle physiology who received procedural deep sedation performed by intensivists between January 2013 and July 2020. Sedative and analgesic medications used were not protocolized and were up to the discretion of the physician performing the sedation.

\section{Results:}

Thirty-three sedations were performed on 27 unique patients. The median age was 3.7 years $\left(25^{\text {tho }} \%, 75^{\text {th }} \%: 2.1,15.6\right)$. The majority of procedures, $88 \%(29 / 33)$, were done on children with Fontan physiology. The remaining 12\% (4/33) were status-post Glenn or hemi-Fontan procedure. The underlying primary cardiac defect was hypoplastic left heart in $52 \%(17 / 33)$ of procedures.

There were 24 chest tube placements and 9 cardioversion procedures; there were no procedural complications.

Ketamine alone [median dose $1.52 \mathrm{mg} / \mathrm{kg}$ (range 0.79-3.70)], ketamine [median dose 0.98 $\mathrm{mg} / \mathrm{kg}$ (range 0.13-2.12)] with propofol [median dose $2.27 \mathrm{mg} / \mathrm{kg}$ (range 0.66-3.77)], and ketamine [median dose $1.50 \mathrm{mg} / \mathrm{kg}$ (range 0.42-3.02)] with morphine [median dose $0.06 \mathrm{mg} / \mathrm{kg}$ (range 0.03-0.20)] were the most common sedation protocols used.

There were minor complications during four procedures. One patient with transient hypotension, two had desaturation, and the fourth had desaturation and apnea. The patients with desaturation received nasal cannula oxygen delivery. The patient with apnea had bag mask valve ventilation. No intervention was reported for hypotension.

\section{Conclusions:}

Procedural deep sedation can be safely and effectively administered to single ventricle patients by intensivist led sedation teams. 